\title{
A Telehealthcare System to Care for Older People Suffering from Metabolic Syndrome
}

\author{
Kevin C. Tseng 1 , Chien-Lung $\mathrm{Hsu}^{2}$, and $\mathrm{Yu}-\mathrm{Hao}$ Chuang ${ }^{3}$ \\ ${ }^{1}$ Product Design and Development Lab, Chang Gung University, \\ Taoyuan, Taiwan \\ ${ }^{2}$ Department of Information Management, Chang Gung University, \\ Taoyuan, Taiwan \\ ${ }^{3}$ Department of Information Management, National Central University, \\ Taoyuan, Taiwan \\ ktseng@pddlab.org, clhsu@mail.cgu.edu.tw, \\ 954403004 @CC.ncu.edu.tw
}

\begin{abstract}
As individuals live longer, the social structure is rapidly changing, resulting in problems such as shortages of medical resources and reduction of quality in healthcare services. Hence, this paper presents a telehealthcare system for user-friendly and long-term healthcare applications for older people suffering from metabolic syndrome. The system can transfer and manage medical data at a distance via a wireless sensor network. The integration of these technologies allows personal stand-alone vital data to become a total telehealthcare solution in home-level care.
\end{abstract}

Keywords: Telehealthcare system, metabolic syndrome, older person.

\section{Introduction}

With the aging of the world population, the United Nations reports that is 21 percent of the world's proportion will be over the age of 60 by 2050 [1]. As in most other countries, the proportion of older people are increasing every year in Taiwan due to decreased birth rates and increased longevity. The proportion of those 65 years and older in Taiwan was approximately $10.2 \%$ in 2007 [2], and is expected to rise $36.71 \%$ in 2050 [3]. Average life expectancy has increased from 76.45 years in 2000 [4] to 78.54 years in 2008 [5].

As individuals live longer, the social structure is rapidly changing, resulting in problems such as shortages of medical resources and reduction of quality in healthcare service. In addition, many older people have at least one or two chronic diseases. Consequently, governments and researchers around the world have to seriously consider health issues [6]. One of the key issues is considering how to look after older persons' health, to keep them healthier, maintain their functions and independence, and improve their quality of life.

There are some studies regarding distributed health care [7-11]. However, many discuss the algorithms and technologies involved in maintaining the capability and 
functionality of distributed healthcare systems, but less has been done to determine to what extent users understand their capacities and functionalities. While it is crucial to improve the capacities and functionality of any healthcare system, a highly advanced system is likely to be rejected by users if it does not fulfill their expectations. The importance of other factors contributing to the acceptance of a telehealthcare system should not be neglected [12]. It is important to investigate users' needs and requirements before designing new systems so true user needs can be satisfied, allowing those systems to have a better chance of gaining user acceptance and adoption. Consequently, there are few systems available for thorough evaluation. In addition, we lack a distributed healthcare model for scientific evaluation from different perspectives (clinical, technical, economic, social, legal, etc.) requiring a multidisciplinary approach. Therefore, a telehealthcare system to care for older people suffering from metabolic syndrome has been proposed.

For elderly individuals suffering from metabolic syndrome, the proposed system provides a user-friendly solution through an integrated telehealthcare environment. Medical data is transmitted and managed at a distance using WSNs (wireless sensor networks). The integration of these technologies allows personal stand-alone vital data to become a total telehealthcare solution in home-level care. The remainder of this paper is organized thus: section 2 described metabolic syndrome. A telehealthcare system is proposed in section 3 and a system validation method is described in section 4. Finally, some conclusions are provided in section 5 .

\section{Metabolic Syndrome}

Metabolic syndrome has received increased attention in the past few years. The syndrome is a constellation of interrelated risk factors of metabolic origin-metabolic risk factors - that appear to directly promote the development of atherosclerotic cardiovascular disease (ASCVD) [13]. Patients with metabolic syndrome have a twofold increased risk of mortality from coronary heart disease and an increased risk for developing type 2 diabetes mellitus [14]. Another set of conditions, the underlying risk factors, give rise to the metabolic risk factors. Understanding how to identify and address the problems of metabolic syndrome can make a significant difference in an older person's health. An older person can realize significant improvements in his or her health status when metabolic syndrome is identified and the problems associated with it are addressed.

In the past few years, several expert groups have attempted to set forth simple diagnostic criteria to be used in clinical practice to identify patients who manifest the multiple components of metabolic syndrome. These criteria have varied somewhat in specific elements, but in general they include a combination of both underlying and metabolic risk factors [15]. Table 1 lists three criteria of metabolic syndrome from three different organizations.

Due to the increasing number of older people with chronic diseases, there are many studies based on different network technologies that propose various designs and applications for telehealthcare service systems [16]. However, most of those systems address their particular situation and specific applications, which does not consider in detail the actual needs of users. 
Table 1. The Criteria of Metabolic Syndrome

\begin{tabular}{|c|c|c|c|}
\hline Factor & WHO 1999 & ATP III * 2001 & $\begin{array}{l}\text { National Health Council, } \\
\text { Taiwan } 2007\end{array}$ \\
\hline Obesity & $\begin{array}{l}\text { WHR: } \\
\text { Male }>0.9 \\
\text { Female }>0.85 \\
\text { BMI }>30\end{array}$ & $\begin{array}{l}\text { Abdominal circumference : } \\
\text { Male } \geqq 102 \mathrm{~cm} \\
\text { Female } \geqq 88 \mathrm{~cm}\end{array}$ & $\begin{array}{l}\text { Abdominal circumference : } \\
\text { Male } \geqq 90 \mathrm{~cm} \\
\text { Female } \geqq 80 \mathrm{~cm}\end{array}$ \\
\hline Triglyceride & $\geqq 150 \mathrm{mg} / \mathrm{dl}$ & $\geqq 150 \mathrm{mg} / \mathrm{dl}$ & $\geqq 150 \mathrm{mg} / \mathrm{dl}$ \\
\hline Blood pressure & $\begin{array}{l}\mathrm{SBP} \geqq 140 \mathrm{mmHg} / \\
\mathrm{DB} \mathrm{P} \geqq 90 \mathrm{mmHg}\end{array}$ & $\begin{array}{l}\mathrm{SBP} \geqq 130 \mathrm{mmHg} / \\
\mathrm{DBP} \geqq 85 \mathrm{mmHg}\end{array}$ & $\begin{array}{l}\mathrm{SBP} \geqq 130 \mathrm{mmHg} / \\
\mathrm{DBP} \geqq 85 \mathrm{mmHg}\end{array}$ \\
\hline Glucose & IGT, IFG, or DM & $\mathrm{FG} \geqq 110 \mathrm{~m} \mathrm{~g} / \mathrm{dl}$ & $\mathrm{FG} \geqq 100 \mathrm{~m} \mathrm{~g} / \mathrm{dl}$ \\
\hline $\begin{array}{l}\text { HDL } \\
\text { Cholesterol }\end{array}$ & $\begin{array}{l}\text { Alb/Cr ratio }>30 \mathrm{mg} / \mathrm{g} \\
\mathrm{AER}>20 \mu \mathrm{g} / \mathrm{min}\end{array}$ & $\begin{array}{l}\text { Male } 40 \mathrm{mg} / \mathrm{dl} \\
\text { Female }<50 \mathrm{mg} / \mathrm{dl}\end{array}$ & $\begin{array}{l}\text { Male }<40 \mathrm{mg} / \mathrm{dl} \\
\text { Female }<50 \mathrm{mg} / \mathrm{dl}\end{array}$ \\
\hline
\end{tabular}

To achieve the goal of telehealthcare, and to look after older people suffering from metabolic syndrome, we propose a telehealthcare system to integrate stand-alone medical devices into WLAN networks and use it to construct a telehealthcare network infrastructure.

\section{A Telehealthcare System}

The section addresses how the proposed system is developed according to the analysis on the needs of distributed health care at home. The system not only receives physiological signals, but also transfers physiological data through the wireless network to the back-end health management server, so that complete and continuous personal physiological data can be recorded.

A three-layer structure of user base, function base, and data base was proposed. The user-based layer has two types of interfaces, web-based and client-based. On the web-based interface, users can connect to the server via HTTP protocol and need only a general browser to log into the system; on the client-based interface, users can log in with a RFID card provided by the study. The function-based layer consists of the application system, external program interface, and system interface. The system interface shows animated scenarios based on physiological data that the intellectual medical apparatus receives. The data-based layer is an information database that records vital sign parameters that the intellectual medical apparatus receives as personal information of users, voice messages, etc.

The programming languages of the system implementation are ASP.NET and PHP, built on Windows Server 2005 with IIS as the site server and Microsoft SQL as the database. The operating environment of the system mainly consists of the user interface, web server, and database. The system development information is summarized in Table 2.

In response to user needs, this study develops a telehealthcare system for long-term care at home. The system is divided into four modules: communication transmission module, membership management module, information integration module of health education for the elderly, and personal health management module. 
Table 2. The System Development Information

\begin{tabular}{||l|l|}
\hline Development language & ASP.NET, PHP \\
\hline \multirow{4}{*}{ System environment } & Windows Server 2005 \\
\cline { 2 - 2 } & Microsoft .NET Framework \\
\cline { 2 - 2 } & IIS Server \\
\cline { 2 - 2 } & Microsoft SQL Server \\
\hline Development software & Microsoft Visual Studio 2005, Flash \\
\hline
\end{tabular}

The system provides different logged identifications with different authorities. The authority design is depicted below (Figure 1):

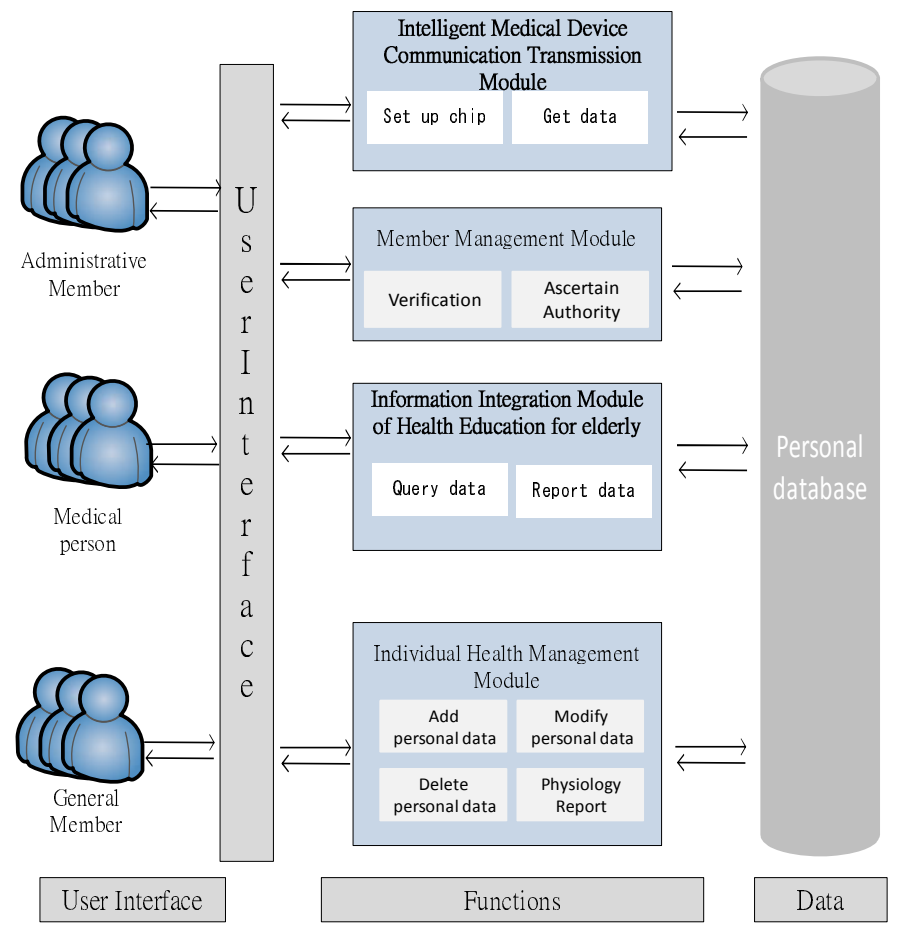

Fig. 1. The System Structure of a Telehealthcare system

Communication Transmission Module. The study employs the Wireless Sensor Network (MSP430) module, developed by the National Taiwan University (NTU) [17]. The structure of the NTU developed wireless sensor networks hardware platform is comprised of three levels: base node, advanced node, and universal gateway. A PC can thus be easily connected via USB interface, and the computer hardware does most of the work transmitting instructions and data. Since the computer has direct power, there is no need to consider energy issues such as nodes [18]. The chip is installed 
in the medical apparatus so that when a user uses the physiological data measuring instrument, the information will be sent to the system chip via the installed chip and saved. The information integration module of health education for the elderly then comprehends collected user parameters and accordingly provides information about health education on the system. The system also stores data as XML format via XML Parser to facilitate the physiological data acquisition for the elderly who log in on the web-based system.

Membership Management Module. The module mainly verifies users' identities and sends a successful login message to users. The RFID card specification that the study employs is depicted in Table 3. Through RFID communication technology, user physiological measurements and card identifiers can be used to send user voice messages.

Table 3. RFID Reader Specification

\begin{tabular}{|l|l|}
\hline \multicolumn{2}{|l|}{ Communication specification } \\
\hline Frequency & $2.4 \mathrm{GHz}-2.5 \mathrm{GHz}$ ISM microwave band \\
\hline Channel & 125 \\
\hline Channel bandwidth & $1 \mathrm{MHz}$ \\
\hline Modulation & GFSK \\
\hline Identification method & Omnidirectional identification \\
\hline Recognition distance & Within $80 \mathrm{~m}$ \\
\hline Recognition speed & Within $80 \mathrm{~m} /$ second \\
\hline Anti-collision & Simultaneously identity more than 100 cards \\
\hline \multicolumn{2}{|l|}{ Interface specification } \\
\hline Ethernet & $10 \mathrm{M} / 100 \mathrm{M}$ Ethernet (RJ-45) \\
\hline RS-232 & RX, TX \\
\hline Communication protocol & TCP \\
\hline Communication speed & $9600 \mathrm{Bps} \sim 115200 \mathrm{Bps}$ \\
\hline \multicolumn{2}{|c|}{ Power requirement } \\
\hline Power & $<120 \mathrm{~mA}, 7.5 \mathrm{~V}$ \\
\hline RF power & $0 \mathrm{dBm}$ \\
\hline Sensitivity & $-90 \mathrm{dBm}$ \\
\hline
\end{tabular}

Information Integration Module of Health Education for the Elderly. There are two types of system displays, one on the interactive system (Figure 2) and the other on the web-based page through the network login system. On the interactive system display, the physiological data is divided into three levels based on the diagnosis mechanism of metabolic syndrome (Figure 3): sunny day (normal), cloudy day (cautious), and rainy day (severe) to show the current physical conditions of users. On the intuitive interface, operation is easy, and the context interface is supplemented to notify the physiological conditions of users. In addition, the system provides web 
functions to assist web users in logging in via the general Internet or mobile phones to view messages that families leave and their physical records. Through detailed information from the electronic records, clinical staff or family members get to know the health conditions of their patients or elderly family members. Moreover, electronic records instead of handwritten ones not only allow the clinical staff to manage the patients easily, but also can be sent to hospitals to assist in diagnoses and to reduce the possibility of tampering with physical records (see Figure 4).

Personal Health Management Module. The module provides the medical centre with the personal information of the elderly patient and also allows authorized clinical staff to add, delete, and modify such information based on the clinical diagnosis.

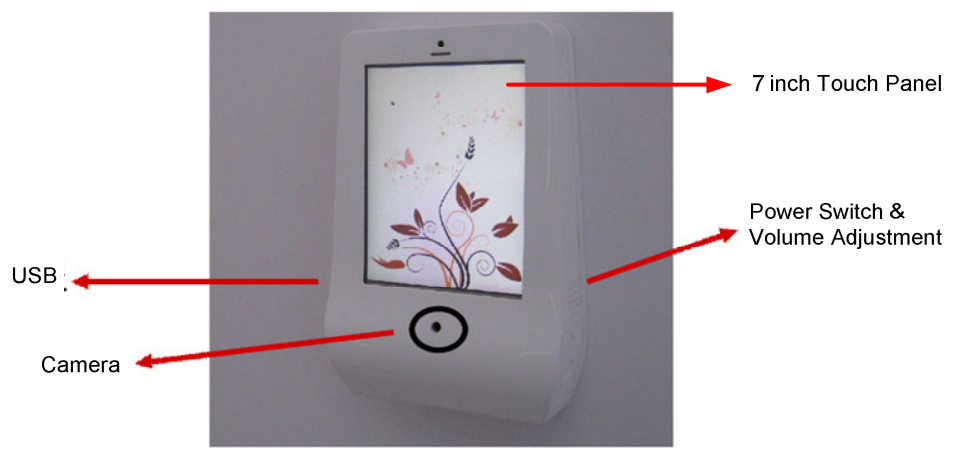

Fig. 2. The Interactive Platform

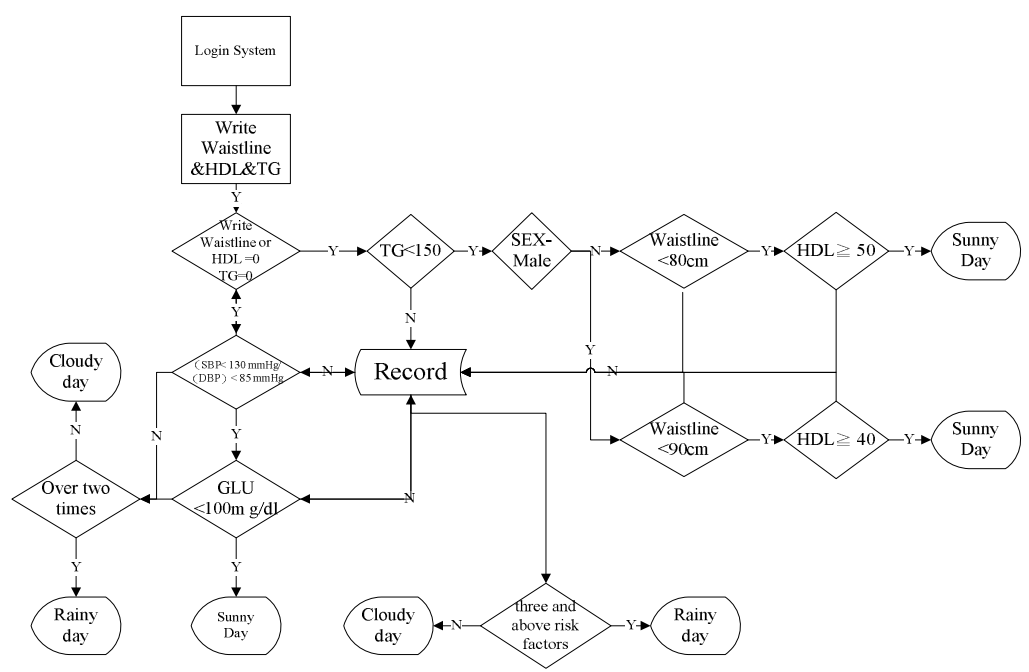

Fig. 3. The Diagnosis Mechanism of Personal Health Management 
血壓標準值：收蛨壓與舒張壓在120到80之間。

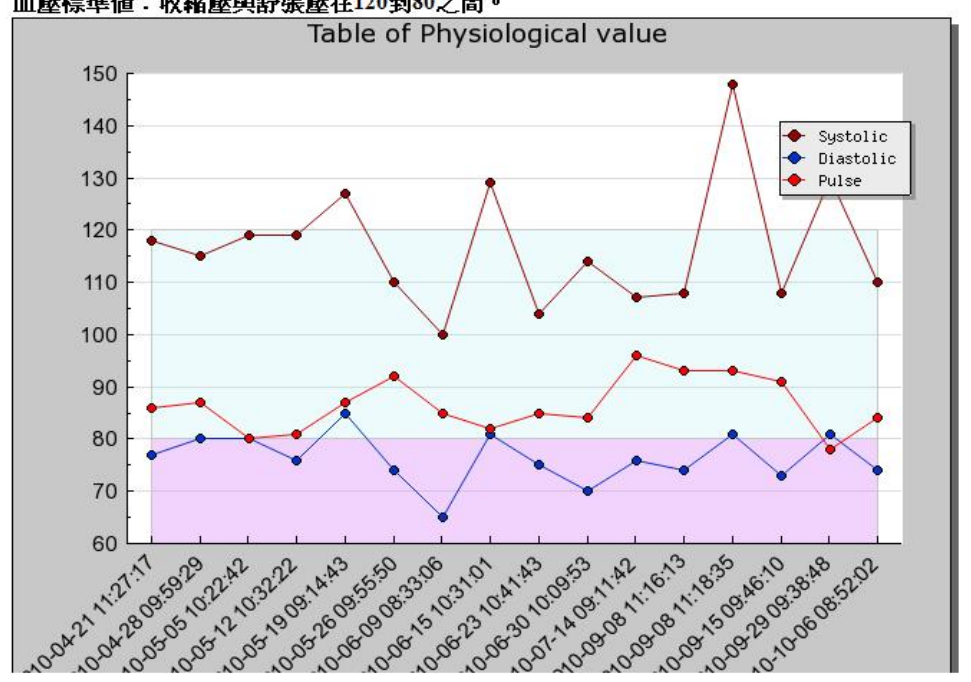

Fig. 4. The Record of Blood Pressure

\section{System Validation}

An experiment was conducted to test the effectiveness of the system. We set up two different environments, an area without any furniture and an area with furniture (see Figure 5). The system could send and receive wireless signals at distances of 3, 6, 7, $8,9 \mathrm{~m}$ and $10 \mathrm{~m}$ to connect to the blood pressure devices. We found that better transmission quality was offered in $7 \mathrm{~m}$ or less. Regardless of whether the area was furnished, data transfer had up to $100 \%$ integrity (see Table 4).

According to the results, the transmission quality was affected by some obstructions such as furniture when the signal path was long. Thus, device placement within $7 \mathrm{~m}$ should be considered when implementing a system in a home in which there are many chairs and tables.
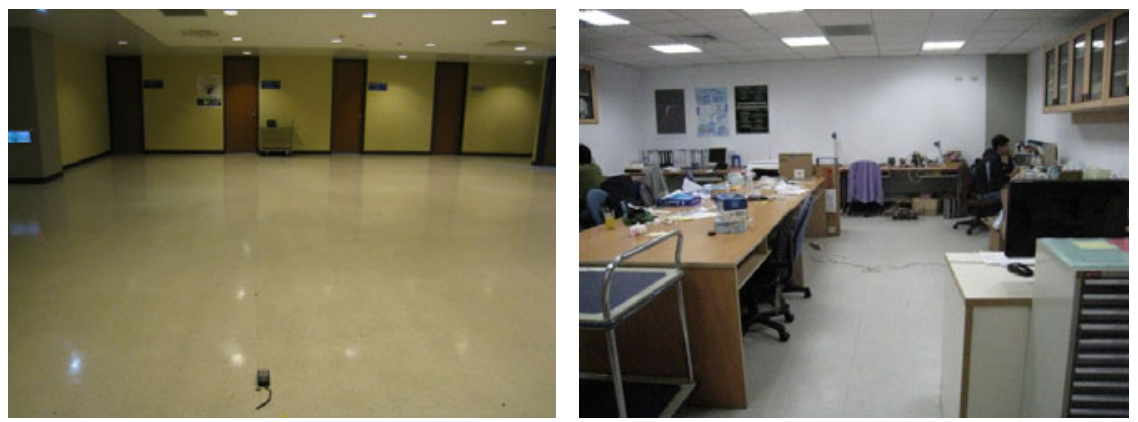

Fig. 5. The Experimental Environment 
Table 4. Transmssion Quality in Distance

\begin{tabular}{|l|l|}
\hline Distance (m) & Completion Rate \% \\
\hline 0 & $100 \%$ \\
\hline 3 & $100 \%$ \\
\hline 6 & $100 \%$ \\
\hline 7 & $100 \%$ \\
\hline
\end{tabular}

The experiment showed the system could maintain good data transmission with an effective radius of $7 \mathrm{~m}$, which is the size of a living room size in an ordinary home. Hence, the system can be set in the living room or bedroom to help an older person suffering from metabolic syndrome record his/her physiological data and send it to his/her family doctor.

\section{Conclusion}

In this paper, we proposed and implemented a telehealthcare system integrating standalone medical devices in the form of a ubiquitous network providing a service platform to provide older persons suffering metabolic syndrome with physiological monitoring. As shown by the experiment, the system could maintain good data transmission with an effective radius of $7 \mathrm{~m}$, which is the size of a living room size in an ordinary home.

The system is a health management system. Professional medical personnel evaluate the daily or weekly measurements of every family member suffering from metabolic syndrome managed by our system. As for family members who upload physiological signals normally, our system will calculate the health curve for each case. This system will subsequently classify the health status of the family member as "sunny", "cloudy", or "rainy" according to the variation of his/her physiological signals and transmit the information to family members, the member's relatives, and the health manager for health-care management.

Acknowledgment. This work was supported in part by the National Science Council, Taiwan, ROC under Grant NSC99-2410-H-182-034 and the Chang Gung Medical Foundation, Taiwan, ROC under Grant CMRPD290061.

\section{References}

1. UN, World population aging: 1950-2050, United Nations, New York ST/ESA/SER.A/207 (2001)

2. CEPD, Taiwan Statistical Data Book (2008)

3. CEPD. Population Projections for Taiwan Area: Medium Variant 2006-2051

4. Ministry of the Interior, 2000 Abridged life table in Taiwan area - Overall, Department of Statistics, 1st edn. Ministry of the Interior, Taipei (2000)

5. Ministry of the Interior, 2008 Abridged life table in Taiwan area - Overall, Department of Statistics, 1st edn. Ministry of the Interior, Taipei (2008) 
6. Koch, S.: Home telehealth-current state and future trends. International Journal of Medical Informatics 75, 565-576 (2006)

7. Lee, H.J., et al.: Ubiquitous healthcare service using Zigbee and mobile phone for elderly patients. International Journal of Medical Informatics 78, 193-198 (2009)

8. Lorenz, A., Oppermann, R.: Mobile health monitoring for the elderly: Designing for diversity. Pervasive and Mobile Computing 5, 478-495 (2009)

9. Milenkovic, A., et al.: Wireless sensor networks for personal health monitoring: Issues and an implementation. Computer Communications 29, 2521-2533 (2006)

10. Bielli, E., et al.: A wireless health outcomes monitoring system (WHOMS): Development and field testing with cancer patients using mobile phones. BMC Medical Informatics and Decision Making 4, 7 (2004)

11. Su, C.J.: Mobile multi-agent based, distributed information platform (MADIP) for widearea e-health monitoring. Computers in Industry 59, 55-68 (2008)

12. Hsu, C.-L., et al.: An investigation of the behavior of middle-aged people in using telehomehealth services. Social Behavior and Personality: An International Journal (in press)

13. Isomaa, B.: A major health hazard: The metabolic syndrome. Minireview 73, 2395-2411 (2003)

14. Eriksson, J., et al.: Exercise and the metabolic syndrome. Diabetologia 40, 125-135 (1997)

15. Scott, C.L.: Diagnosis, prevention, and Intervention for the metabolic syndrome. Am. J. Cardiol. 92, 35i- 42i (2003)

16. Chan, M., et al.: A review of smart homes - Present state and future challenges. Computer Methods and Programs in Biomedicine 91, 55-81 (2008)

17. Pan, J.-J., et al.: Wireless sensor network platform and application. Science Development 5 (2010)

18. Kao, M.K.: WSN Platform - Super Node (September 29, 2010),

http://www.wsnc.ntu. edu.tw/Files/SuperNode.pdf 